\title{
Yod
}

Revue des études hébraïques et juives

$17 \mid 2012$

La presse écrite au Moyen-Orient

\section{Hazefirah, premier journal de langue hébraïque en Pologne}

Hazefirah, the First Hebrew Periodical in Poland

$$
\text { הצפירה, כתב העת העברי הראשון בפולין }
$$

\section{Lily Perlemuter}

\section{OpenEdition}

\section{Journals}

Édition électronique

URL : https://journals.openedition.org/yod/1580

DOI : $10.4000 /$ yod. 1580

ISSN : 2261-0200

\section{Éditeur}

INALCO

Édition imprimée

Date de publication : 30 juin 2012

Pagination : 53-65

ISBN : 978-2-85831-200-9

ISSN : 0338-9316

\section{Référence électronique}

Lily Perlemuter, « Hazefirah, premier journal de langue hébraïque en Pologne », Yod [En ligne], 17 | 2012, mis en ligne le 30 octobre 2012, consulté le 08 juillet 2021. URL : http://journals.openedition.org/yod/ 1580 ; DOl : https://doi.org/10.4000/yod.1580

Ce document a été généré automatiquement le 8 juillet 2021.

\section{(ब) $(1) \Theta$}

Yod est mis à disposition selon les termes de la Licence Creative Commons Attribution - Pas d'Utilisation Commerciale 4.0 International. 


\section{Hazefirah, premier journal de langue hébraïque en Pologne}

Hazefirah, the First Hebrew Periodical in Poland

הצפירה, כתב העת העברי הראשון בפולין

\section{Lily Perlemuter}

1 Cette étude, consacrée au journal Hazefirah ${ }^{1}$ et plus particulièrement à la place qu'il accorde à la littérature hébraïque, comporte deux axes : son histoire et l'écho qu'il fait de la célébration des soixante-quinze ans du très grand écrivain de langues hébraïque et yiddish, Mendele Mokher Sefarim². C'est à partir de ces deux axes que nous mettrons en relief le rôle de la littérature dans le journal et l'importance de la vie littéraire dans la communauté juive de Pologne.

2 Hazefirah, premier journal de langue hébraïque en Pologne, est fondé par Haïm Zelig Slonimski (1810-1904) qui en est également le rédacteur en chef. Le premier numéro du journal, qui au début paraît chaque semaine, sort le 4 février 1862, à Varsovie, avec le sous-titre :

מכתב עתי, משמיע חדשות בקרב עם ישרון מכל הדברים הנוגעים להם בעניני

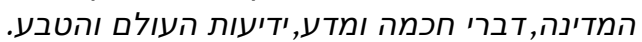

Périodique publiant des nouvelles concernant le peuple juif et ayant trait à des sujets politiques, au savoir et à la science, à la connaissance du monde et de la nature.

Ce sous-titre indique d'emblée les deux principaux objectifs poursuivis: traiter de l'actualité et fournir aux lecteurs des connaissances sur les sciences -- tout particulièrement les sciences naturelles et technologiques. Ce deuxième objectif est caractéristique de l'époque. En effet, le mouvement de la Haskala, les «Lumières juives ", s'est donné pour but de transformer le peuple juif, d'en faire un peuple comme les autres en évitant son cantonnement à la culture juive et en l'ouvrant au savoir universel.

4 Lorsque le journal commence à paraître, il doit tenir largement compte du public auquel il s'adresse : ses lecteurs, en Pologne, sont pour la plupart des hassidim ${ }^{3}$ très attachés à la religion et à la tradition. Le ton doit donc être modéré et démontrer qu'il n'y a pas d'antagonisme entre le savoir universel et le respect des préceptes religieux. 
Quant au traitement des nouvelles politiques, il doit tenir compte de la censure du gouvernement polonais.

5 Il faut noter que le mot littérature ne figure pas dans le sous-titre. En effet, au début de sa parution, Hazefirah ne consacre à la littérature qu'une place très réduite, voire inexistante.

6 Shalom Yaakov Abramovitch, plus connu sous son nom de plume Mendele Mokher Sefarim (qu'il ne porte pas encore à l'époque), est un des tout premiers collaborateurs. À partir du 26 février 1862, il publie une série d'articles sur les oiseaux, puis à partir du mois d'avril une dizaine d'articles sur la chimie ${ }^{4}$. Il est rejoint durant la première année par Y. L. Gordon (1830-1892), le porte-drapeau de la Haskala en Lituanie, et par Kalman Shulman (1819-1899) .

7 Le 24 juillet 1862 (après six mois d'existence), le journal annonce qu'il interrompt sa parution. Le rédacteur en chef explique qu'il doit quitter Varsovie et qu'il n'a pas trouvé de remplaçant. L'interruption durera douze ans, d'août 1862 à juillet 1874.

Lorsqu'il renaît le 8 juillet 1874, Hazefirah ne paraît plus à Varsovie mais à Berlin, surtout pour éviter des difficultés administratives. À partir de septembre 1875, il paraîtra à nouveau à Varsovie en gardant la même ligne éditoriale. Il propose des nouvelles de différents pays, des articles scientifiques et une nouvelle rubrique sur les actualités littéraires qui paraît de façon irrégulière. Nahum Sokolov (1859-1936), qui deviendra plus tard rédacteur en chef, commence à y publier. À partir de 1876, on y trouve des nouvelles de Jérusalem dans une rubrique intitulée " Lettres de Jérusalem », sous la responsabilité d'Abraham Moshé Luncz'.

9 Le 14 novembre 1882, le sous-titre du journal change et devient moins explicite: ח "Périodique publiant des nouvelles».

10 Il est intéressant de souligner l'attitude de Hazefirah vis-à-vis de la solution nationale. Dans les années 1880, le journal propose des descriptions de la vie en Palestine en mettant l'accent sur les conditions difficiles de la vie des Juifs. Même après les pogromes de 1881 dans le sud de la Russie, le journal ne voit pas dans la Palestine une solution pour les Juifs.

11 Après le numéro du 5 janvier 1886, le journal cesse de paraître pendant deux mois. Lorsqu'il reprend sa parution le 13 avril 1886, c'est désormais non plus un hebdomadaire mais un quotidien portant le sous-titre: Périodique politique, scientifique et littéraire, paraissant tous les jours ». Les noms des rédacteurs en chef, Slominski et Sokolov, figurent au bas de la troisième page.

12 C'est à partir de cette date que la partie littéraire prend de l'ampleur avec divers articles comme "L'amour des savants chrétiens pour l'hébreu » ou un poème d'Ibn Gabirol. D'autres rubriques, telle דברי סופרים Paroles d'écrivains » en 1887, voient le jour.

13 Le 25 septembre 1888 , le journal annonce qu'il cesse sa parution pendant quelques jours. Il est en fait fermé par la censure et l'interruption durera onze semaines.

14 Le journal publie aussi des histoires sous forme de feuilletons qu'on retrouve semaine après semaine. Parmi les grands écrivains qu'on peut lire en 1888, il faut notamment citer Mikha Yosef Berditchevsky (1865-1921). À cette époque, le journal craint d'aborder les sujets politiques et c'est une des raisons pour lesquelles il approfondit et 
multiplie les rubriques littéraires comme שיחות בעולם הספרות ה Conversations dans le monde littéraire » ou בשוק הספרים והסופרים Au marché des livres et des auteurs ».

Le 13 janvier 1896, le sous-titre du journal change et devient " Périodique sur la politique, les sciences, la littérature et tout ce qui concerne les affaires juives ». Le journal réitère son intérêt pour la littérature. À partir de cette époque, de nombreux écrivains publieront poèmes et nouvelles -- par exemple, le 4 décembre 1896, la nouvelle Yaakov le gardien de Mordekhaï Zeev Feuerberg (1874-1899) et le poème Ne pleurez pas le mort de Y. L. Peretz (1852-1915) ; le $1^{\text {er }}$ juin 1900, le poème de Ouri Nissan Gnessin (1879-1913) Le Don de la Thora ainsi que des nouvelles de Shalom Asch (1880-1957). Les articles consacrés à la langue hébraïque se multiplient également, notamment à partir de janvier 1900, une série d'articles signés Shimon Bernfeld (1860-1940) sur l'histoire de la recherche dans le domaine de la langue hébraïque. On trouve aussi des traductions de la littérature française, comme des nouvelles d'Émile Zola et de Guy de Maupassant. Sokolov écrit un certain nombre d'articles sur la littérature hébraïque : «Les chemins de la littérature hébraïque et ses problèmes ». Durant ces années, le journal publie des auteurs qu'on a l'habitude de lire dans Hamelitz ${ }^{7}$, comme Y. L. Peretz qui signe du nom de Ben Tamar et Yosef Klausner (1874-1958) qui écrira d'ailleurs beaucoup par la suite.

L'attitude du journal envers le mouvement sioniste est intéressante. Au début, Sokolov critique Herzl et pense que le premier congrès sioniste n'aura pas de succès. Sa participation en tant que journaliste à ce premier congrès marquera un tournant dans son attitude vis-à-vis du sionisme politique. En 1900, le journal devient presque le porte-drapeau du mouvement sioniste. Les abonnements sont nombreux et le journal commence à prendre la place de Hamelitz comme principal journal hébreu.

Le 25 décembre 1903, un véritable supplément littéraire hebdomadaire paraît pour la première fois. Il comprend seize pages avec des photographies et des dessins et paraîtra pendant un an. Durant cette année et les suivantes, la littérature occupe une place de choix. De nouvelles rubriques voient le jour comme פרורי ספרות «Miettes de littérature » de Mikha Yosef Berditchevsky, par exemple. Dvora Baron (1887-1956), qui a publié pour la première fois dans Hazefirah en 1903, y publie à nouveau en 1904 ainsi que d'autres auteurs importants comme Yaakov Fichman (1881-1958), Zalman Shneour (1887-1959) qui publie poésie et prose et Yaakov Cohen (1881-1960). Le 29 janvier 1904, une partie du journal est consacrée à Eliezer Ben-Yehuda ${ }^{8}$.

Hazefirah cesse de paraître pendant près de cinq ans, du 13 janvier 1906 au 14 octobre 1910. Lorsqu'il recommence à paraitre en 1910, Yosef Klausner et David Frischmann (1859-1922) y sont très actifs. Frischmann publie régulièrement "Chroniques de mon voyage en Palestine». Fishel Lachover (1883-1947), qui deviendra le rédacteur en chef de la partie littéraire jusqu'en 1918, publie de très nombreux articles sur des écrivains de langue hébraïque comme Haïm Nahman Bialik (1873-1934), Shaoul Tchernikhowsky (1875-1943), Moshe Lilienblum (1843-1910), Nahman Krokhmal (1785-1840) et bien d'autres. Lachover publie parallèlement les œuvres de nombreux auteurs importants: Tchernikhowsky, Moshe Smilansky (1874-1953), Mendele Mokher Sefarim, Shmuel Yosef Agnon (1887-1970) et Bialik (l'essai Loi et légende et la nouvelle La Trompette honteuse).

19 En 1914, Hazefirah pour les enfants commence à paraître au rythme d'une fois par mois. 
Après une interruption de la parution de décembre 1916 à juin 1917, Hazefirah redevient un hebdomadaire.

À partir de 1919, la place consacrée à la littérature diminue beaucoup au profit de celle consacrée au sionisme et à la situation en Palestine. En 1919, le journal reprend le rythme d'une parution quotidienne. Les vingt années qui le séparent de sa disparition définitive en 1931 comportent des interruptions dans sa parution. Les problèmes sont à la fois d'ordre financier et rédactionnel, de nombreux collaborateurs du journal étant partis s'installer en Palestine.

\section{Hazefirah fête les soixante-quinze ans de Mendele Mokher Sefarim}

Le numéro du 6 janvier 1911 comprend six pages dont la presque totalité est consacrée à l'anniversaire de Mendele Mokher Sefarim. Il comporte une biographie de ce dernier et l'annonce faite par Haïm Nahman Bialik des festivités organisées en l'honneur du "prince de la littérature hébraïque et yiddish respecté et aimé dans toute la diaspora juive ». Des réceptions, un banquet et des conférences honorent, d'après Bialik, non seulement l'écrivain, mais aussi toute la littérature hébraïque. Les journaux des jours suivants lui sont également en grande partie dédiés. Tous rendent hommage à celui qu'on surnomme « le grand-père de la littérature et de la langue hébraïques ».

On peut classer toutes ces publications en trois groupes :

- des articles rédigés en l'honneur de l'écrivain par ses collègues ou ses disciples qui relatent souvent des histoires vécues avec Mendele ;

- des informations envoyées d'Odessa où l'anniversaire est célébré, par un journaliste dépêché sur place et qui reprennent des passages des discours prononcés en l'honneur de l'écrivain ;

- des félicitations adressées à l'écrivain par des organisations et des associations.

À travers ces témoignages, c'est un grand écrivain de langues hébraïque et yiddish qui se dessine, le créateur d'un nouveau style en hébreu et une personnalité très attachante.

des articles (notamment ceux du grand auteur yiddish Shalom Aleikhem ${ }^{9}$, mais aussi ceux de Yaakov Fichman et de David Frischmann) met l'accent sur un aspect différent de l'art de l'écrivain et sur un trait de sa personnalité.

Shalom Aleikhem intitule son article du 6 janvier 1911 «Autodafé » et ajoute le soustitre : « en l'honneur du grand-père Mendele Mokher Sefarim par son premier petit-fils Shalom Aleikhem ». D'après lui, il a été le premier à surnommer Mendele "grandpère », et Mendele à l'appeler "petit-fils». L'article est écrit avec l'humour qui caractérise Shalom Aleikhem. Celui-ci raconte un épisode de sa jeunesse et de ses débuts d'écrivain. Âgé à l'époque d'une vingtaine d'années, Shalom Aleikhem est très influencé par Mendele. Il a écrit un livre, une sorte d'allégorie, et désire le montrer au maître pour avoir son approbation. Après bien des hésitations, Shalom Aleikhem invite Mendele dans sa résidence d'été. Les deux hommes s'installent pour que Mendele écoute la lecture du roman. Avant de commencer, ce dernier demande à Shalom Aleikhem: "Dis-moi qu'as-tu voulu, qu'as-tu cherché en écrivant ton roman?", "Qu'ai-je voulu? Qu'est-ce que ça veut dire? » demande Shalom Aleikhem. «Oui », insiste Mendele, « un écrivain qui commence à écrire doit se poser cette question.» C'était pour Shalom Aleikhem comme la découverte de l'Amérique. Cela faisait cinq ans 
qu'il écrivait et il ne s'était jamais posé la question. Que peut vouloir un écrivain ? Il veut écrire! Shalom Aleikhem commence alors à lire son roman devant Mendele qui a les yeux fermés, l'air très concentré. Subitement, ce dernier l'interrompt et demande si les cuisinières ont commencé à préparer le déjeuner. Shalom Aleikhem pense alors que Mendele a faim et propose de lui apporter quelque chose à manger. Mais Mendele rétorque qu'il veut juste savoir si le poêle est déjà allumé. «Oui, bien sûr, le poêle est déjà allumé ", lui répond Shalom Aleikhem. "Alors », dit Mendele, " sois gentil, prends ce cahier avec ton roman et jette-le au feu. Ce n'est pas le genre de littérature pour lequel tu es doué. » Depuis, raconte Shalom Aleikhem, il a rencontré Mendele maintes fois et ils ont souvent parlé d'écriture, de style mais n'ont jamais évoqué l'épisode de cet autodafé. Shalom Aleikhem n'a donc jamais eu l'occasion de remercier Mendele pour la leçon qu'il a reçue. Il sait à présent combien il est important que le poêle soit allumé quand un auteur termine d'écrire un livre qui lui semble parfait.

L'article que publie Yaakov Fichman le 6 janvier 1911 a pour titre «Une soirée avec Mendele ». Fichman raconte qu'un soir, rempli de crainte, il rend visite à Mendele. Lui est un jeune auteur et Mendele, une grande personnalité. Lors de leur conversation, Mendele critique les jeunes écrivains qui manquent d'originalité et choisissent tous la même voie. Selon lui, de nombreux domaines de la littérature sont négligés. Mendele raconte à Fichman qu'il souffre d'insomnie. La nuit, il marche dans sa chambre, pense et ses réflexions sont tristes. Il peut se consoler dans le cimetière attenant à sa chambre, où il se promène parmi les tombes. Fichman ne comprend pas ces propos. Mendele ouvre alors la porte d'une grande pièce tapissée de livres de littérature hébraïque ancienne et nouvelle. "Voici notre grand cimetière », dit-il avec tristesse, «c'est là que je m'enfuis la nuit. Lorsque je vois tous ces trésors qui n'intéressent personne, j'ai honte de me plaindre de notre sort. Nous avons de telles richesses. » C'est le rire mêlé de tristesse de Mendele, lorsqu'il désigne la grande bibliothèque en disant " voici le grand cimetière attenant à ma chambre ", que Fichman garde en mémoire.

David Frischmann, dans son article du 6 janvier 1911, montre à quel point l'écriture de Mendele est ancrée dans le réel. Il raconte l'histoire d'un jeune homme qui, voyageant en train, lit un livre dans lequel il est totalement absorbé. Levant les yeux, il voit un pauvre juif qui discute avec son voisin. Il se remet à lire sans même sentir qu'il a interrompu sa lecture. Le train s'arrête à une gare. Le jeune homme lève la tête, regarde une ferme avec des animaux et lorsqu'il se replonge dans sa lecture, il ne sent pas qu'il s'est interrompu. Le spectacle qu'il voit à l'extérieur et le passage du livre ne font qu'un. En regardant dehors, il a l'impression de lire un paragraphe du livre. Le jeune homme continue à lire et, dans les pages, défilent des Juifs misérables, mendiants et colporteurs, des enfants privés d'enfance. Les descriptions sont teintées d'ironie et de moquerie mais aussi imprégnées de larmes et d'une grande empathie. Le jeune homme qui lit le livre, c'est David Frischmann et l'auteur qu'il lit, Mendele Mokher Sefarim.

Frischmann tente aussi de comprendre le génie de Mendele qui ne s'inspire d'aucun de ses prédécesseurs. D'après lui, les œuvres de Mendele comprennent plusieurs éléments essentiels :

- la peinture de la vie des Juifs en diaspora, tous les types de Juifs y figurent ;

- des descriptions de la nature qu'on ne trouve chez aucun autre poète juif de l'époque ;

- l'ironie, la satire et la moquerie envers les Juifs, qui sont toujours accompagnées de gémissements et de pleurs. 
Mendele dans la renaissance nationale. À l'époque du jeune Mendele, la solution nationale n'était pas encore envisagée. Mendele n'a donc pu décrire que le pan très sombre de la vie juive. Il a dépeint avec tant d'acuité la situation des Juifs que le peuple a été obligé de se révolter et de chercher une solution. Ses écrits ont ainsi préparé les cœurs à l'idée de la renaissance nationale. Il peut, de ce fait, être considéré comme l'un de ses pères. Il n'y a pas de démonstration plus forte de l'unité de la nation juive que l'œuvre de Mendele. «Ce père de la nouvelle littérature et du nouveau style fait partie des biens nationaux du peuple juif et son anniversaire doit donc être un jour de fête et de joie. Il ne s'agit pas de la fête d'un seul écrivain, il s'agit de la fête de la littérature juive. » devenu célèbre en Pologne? " À cette époque, en Pologne, contrairement à ce qu'on pourrait penser, les lecteurs de langue yiddish sont peu nombreux. Les Juifs polonais ne connaissent pas les premières histoires de Mendele. Même son yiddish est différent de celui parlé par les Juifs polonais. Le mot yiddish קליאטשע «jument» ${ }^{10}$ est, par exemple, un mot qu'ils ne connaissent pas. Et subitement, en Pologne les Juifs et les chrétiens apprennent qu'il y a un écrivain très talentueux qui écrit des histoires en yiddish. C'est grâce à un non-juif que Mendele est devenu célèbre : l'écrivain polonais Klemens Junosza (Szaniawski) ${ }^{11}$. Celui-ci connaît bien les Juifs et leur mode de vie et écrit sur eux. Tous pensent qu'il connaît le yiddish. Un étudiant, natif de Lituanie, admirateur de Mendele, parle de lui à Klemens Junosza qui décide alors de le traduire en polonais. Il le traduit du russe avec l'aide de cet étudiant. Sur le livre, il n'est pas indiqué que le livre a été écrit à l'origine en yiddish. Il est publié en 1885 sous le titre Le Don Quichotte juif, esquisse de la littérature juive par Klemens Junosza. Les Polonais sont surpris d'apprendre que les Juifs ont une littérature qui mérite d'être traduite et ces derniers sont très fiers. Le livre remporte un tel succès qu'une maison d'édition demande à Junosza de traduire un autre livre de Mendele. Junosza choisit Di Klatché, "La Jument", et puisque Mendele est déjà connu des lecteurs polonais, il est mentionné sur la couverture que le livre est de Shalom Yaakov Abramovitch. De nombreux Juifs, notamment des femmes qui ne lisent pas le yiddish, lisent Mendele en polonais. C'est ainsi qu'il devient célèbre en Pologne aussi bien parmi les Juifs que parmi les chrétiens.

33 Les festivités organisées en l'honneur de Mendele Mokher Sefarim sont décrites pendant plusieurs jours par l'envoyé spécial de Hazefirah à Odessa. Elles comprennent une réception au domicile de Mendele, un banquet et une représentation théâtrale. Des discours sont prononcés en hébreu, en russe et en yiddish par Bialik, Klausner, Menahem Ussishkin ${ }^{12}$, Kleinman et d'autres. Mendele répond par un magnifique discours qui provoque un grand enthousiasme. Le héros de la fête est élu à de nombreuses sociétés savantes. Plusieurs délégations arrivent et Mendele reçoit plus de mille télégrammes de félicitations ainsi que des cadeaux.

34

C'est Bialik qui, le premier, prend la parole au nom des associations littéraires. Il offre au vieillard l'ensemble de son œuvre dans une nouvelle édition avec une couverture en argent. Bialik met en exergue dans son discours la contribution de Mendele à la littérature : 
La valeur des perles que vous avez ajoutées aux trésors de notre littérature est inestimable. Lorsque le peuple juif aura trouvé la paix et oublié ses malheurs, il pourra se plonger dans sa littérature et retrouver les générations qui l'ont précédé: des Juifs authentiques, dans leur laideur et leur beauté, dans leurs haillons et leurs vêtements de soie du shabbat, dans leurs joies et leurs peines. Les lecteurs y trouveront peut-être aussi une larme cachée, celle de Mendele $^{13}$.

Bialik insiste aussi sur l'immense contribution de Mendele à la renaissance de la langue hébraïque qu'il a enrichie, tout en lui donnant de nouvelles couleurs et en permettant aux jeunes talents d'avoir un outil à leur mesure. Il met aussi l'accent sur le bilinguisme de Mendele : pour lui, l'auteur de langue yiddish et l'auteur de langue hébraïque sont indissociables.

Yosef Klausner, grand critique littéraire, prend la parole en disant que Mendele n'est pas un auteur au sein d'un peuple inculte. Quatre cents ans de création littéraire le précèdent. Néanmoins, parmi les géants de la littérature et depuis que la littérature profane existe, il n'y a pas eu d'aussi grand artiste que Mendele, même pendant l'âge d'or espagnol.

Menahem Ussishkin fait son allocution en russe. Selon lui, bien que Mendele n'ait jamais fait partie du mouvement des Amants de Sion ${ }^{14}$, il l'a aidé sans en avoir eu vraiment l'intention. La renaissance de la littérature et de la langue hébraïques va de pair avec la renaissance nationale. Les véritables héritiers spirituels de Mendele se comptent parmi les habitants de la terre d'Israël auxquels il n'a pas pensé en écrivant. Ses lecteurs seront les futurs jeunes habitants de la terre d'Israël qui désireront donner un nouvel essor à la littérature hébraïque et accroître son rayonnement.

Mendele répond aux orateurs et insiste sur le fait qu'il ne faut pas avoir peur de la concurrence entre l'hébreu et le yiddish. Lui-même n'aurait pas pu négliger l'une des deux langues. Elles font partie de lui depuis sa plus tendre enfance :

Nos Sages ont dit que le juif a deux âmes: une âme sacrée et une âme profane. L'hébreu est notre âme sacrée, pure, dans laquelle le monde se reflète comme dans un miroir. Elle est vieille comme le monde. L'autre est notre âme profane, celle de la tristesse, de la misère comme l'âme des Juifs des jours ouvrables ${ }^{15}$.

Pour saisir la véritable dimension qu'occupent les festivités dans le journal, il faut insister sur le fait que pendant une dizaine de jours, des félicitations émanant de différentes organisations du monde juif y sont publiées. Quelques exemples peuvent illustrer la teneur des messages :

Les jeunes de la région de Lublin souhaitent à Mendele que, de son vivant, le peuple d'Israël vive en sécurité et qu'il puisse voir sa jument parmi les chars $\left.d^{\prime} I s r a \ddot{e}\right|^{16}$. Les membres $d u$ mouvement Ha-Tehiya ${ }^{17}$ lui souhaitent de pouvoir dépeindre la vie du peuple juif avec des couleurs lumineuses en terre d'Israël. De Lituanie, on lui souhaite d'avoir encore beaucoup d'années pour travailler: "Que Dieu fasse que la belle dame qui est la langue hébraïque soit à votre droite et vous aide à écrire et qu'elle devienne la langue dominante lorsque viendra de Sion la rédemption d'Israël. » Ou encore: "À Mendele, l'homme aimé par la nation, nous souhaitons de voir de ses yeux sa jument paître dans les prés de Sion et de Jérusalem. »

La célébration des soixante-quinze ans de Mendele Mokher Sefarim et la couverture faite par Hazefirah de l'événement soulignent l'importance de cet écrivain dans la littérature hébraïque. Elles démontrent également l'importance de la littérature dans la vie juive et son rôle dans la naissance d'une idée et d'un projet nationaux. Par ailleurs, le fait que Hazefirah consacre une telle place à cet événement prouve aussi sa vocation de prendre une part active à la construction et à la formation d'une nation. 
41 Cette étude n'aborde qu'une infime partie de l'étendue du savoir, de la richesse des idées et de la variété des sujets contenus dans les pages du journal. Hazefirah est également un des témoins majeurs de la vie juive pendant les soixante-dix ans que couvre sa parution. Il s'agit d'une période riche en interrogations, en hésitations, en changements qui transparaissent dans le journal. On peut dire qu'il voit le jour avec les balbutiements de l'ouverture au monde moderne et s'éteint quand la solution nationale et l'indépendance du peuple juif pointent à l'horizon.

\section{NOTES}

1. Le nom signifie en hébreu «l'aurore", d'après Ézéchiel 7, 10: הנה היום הנה באה יצאה הצפירה צץ המטה פרח הזדון " Voici le jour ! Voici qu'elle vient, qu'elle pointe l'aurore ; la verge a fleuri, l'impudence est éclose. » (traduction de la Bible du Rabbinat).

2. Mendele Mokher Sefarim (ce pseudonyme signifie « Mendele marchand de livres »), de son vrai nom Shalom Yaakov Abramovitch (Kopyl, Biélorussie, 1835 - Odessa 1917), est un écrivain de langues yiddish et hébraïque. Il commence par écrire en hébreu puis passe au yiddish qui est, à l'époque, plus adapté à une œuvre réaliste et dont l'audience est plus importante. Il publie en 1864 Le petit homme sous son pseudonyme. En 1873, paraît La Jument. En 1886, il revient à l'hébreu et traduit ses propres œuvres du yiddish. Son hébreu, composé de toutes les strates de la langue, porte souvent le nom d'« hébreu total ». Dans ses nombreuses œuvres, Mendele dépeint la vie misérable des Juifs, colporteurs et mendiants, de manière ironique et satirique mais aussi avec beaucoup d'empathie et d'amour.

3. Juifs appartenant au mouvement religieux populaire fondé au XVIII ${ }^{\mathrm{e}}$ siècle en Podolie par Israël ben Eliezer Baal Shem Tov.

4. Dans un article publié en 1861 dans Hamelitz, il exprime le regret de ne pas avoir de livres en hébreu sur les sciences de la nature. Il annonce qu'il traduit les livres de Lenz. La traduction est publiée en trois volumes : les Mammiferes en 1862, les Volatiles en 1867 et les Reptiles en 1872.

5. K. Shulman a traduit en hébreu Les Mystères de Paris.

6. 1854-1918, auteur, éditeur et géographe de la Palestine.

7. Revue hébraïque éditée à Odessa et à Saint-Pétersbourg entre 1860 et 1904 qui fut le portedrapeau de la Haskala en Russie.

8. Artisan de la renaissance de la langue hébraïque, 1858-1922.

9. Pseudonyme de Shalom Rabinovitch, 1859-1916.

10. Titre du roman allégorique de Mendele Mokher Sefarim.

11. 1849-1898.

12. Homme politique sioniste, $1863-1941$.

13. Publié le 12 janvier 1911.

14. Mouvement présioniste fondé en Russie à la fin du XIX ${ }^{\mathrm{e}}$ siècle dont le but était la création d'un foyer juif en Palestine.

15. Publié le 13 janvier 1911.

16. Cette phrase fait allusion au roman allégorique de Mendele Mokher Sefarim La Jument dans lequel la nation d'Israël prend les traits d'une jument. Il se base sur le verset du Cantique des Cantiques 1, לסוסתי ברכבי פרעה דימיתיך רעיתי A ma cavale parmi les chars de Pharaon je te compare, ma compagne.» 


\section{RÉSUMÉS}

L'étude est consacrée au journal Hazefirah et plus particulièrement à la place qu'il accorde à la littérature hébraïque. C'est le premier journal de langue hébraïque en Pologne, et il parait de 1862 à 1931.

L'étude comporte deux parties : l'histoire du journal et l'écho qu'il fait de la célébration des soixante-quinze ans du très grand écrivain de langues hébraïque et yiddish, Mendele Mokher Sefarim. Le rôle important de la littérature dans le journal et la richesse de la vie littéraire dans la communauté juive de Pologne sont ainsi mis en relief.

This work is about the newspaper Hazefirah, especially the place given to the Hebrew literature. Hazefirah, published from 1862 to 1931, is the first newspaper in Hebrew language in Poland. Our work has two distinct parts: the first one is the history of the newspaper; the second one is about the celebration of the seventy-fifth birthday of the Hebrew and Yiddish writer Mendele Mokher Sefarim. The study shows the important place of literature in the newspaper and the richness of the literary life of the Jewish community in Poland.

\section{INDEX}

Keywords : Hazefirah, Hebrew language, Hebrew literature, Hebrew press, Mendele Mokher Sefarim (1836-1917)

Thèmes : littérature

\section{מילות מפתח}

הצפירה, מנדלי מוכר ספרים, ספרות עברית, עברית, עיתונות עברית:

Mots-clés : Hazefirah, hébreu (langue), littérature hébraïque, Mendele Mokher Sefarim (1836-1917), presse hébraïque 